\title{
CD95/Fas mediates cognitive improvement after traumatic brain injury
}

\author{
Christoph P Beier ${ }^{1,2, *}$, Martina Kölbl ${ }^{2, *}$, Dagmar Beier ${ }^{1}$, Chris Woertgen², Ulrich Bogdahn ${ }^{1}$, \\ Alexander Brawanski ${ }^{2}$ \\ ${ }^{1}$ Department of Neurology, University of Regensburg, Medical School, Universitätsstrasse 84, 93053 Regensburg, Germany; ${ }^{2}$ Depart- \\ ment of Neurosurgery, University of Regensburg, Medical School, Franz-Josef-Strauss Allee 11, 93042 Regensburg, Germany
}

Cell Research (2007) 17:732-734. doi: 10.1038/cr.2007.60; published online 24 July 2007

CD95 (APO-1/Fas)/CD95L (APO-1L/FasL/CD178) is a receptor/cytokine pair of the tumor necrosis factor/nerve growth factor (TNF/NGF) superfamily. Similar to other receptors of this family, activation of CD95/Fas results in the formation of a death-inducing signaling complex (DISC) and subsequent activation of the apoptotic cascade [1]. There is increasing evidence that CD95/Fas also signals survival, proliferation, and migration in CD95/Fas-expressing cells that are resistant to CD95L/FasL-induced cell death $[2,3]$. This non-apoptotic signaling of CD95/Fas is also referred to as "reverse signaling" [3]. In contrast to the apoptotic signaling cascade, the molecular mechanisms resulting in the activation of non-apoptotic pathways are still vague [3].

The physiological role of CD95/Fas expression in the central nervous system is unknown. Both, CD95/Fas and CD95L/FasL have been detected in the brain [4-6], but their spatial and temporal expression patterns change during development $[5,7]$. Mice with defective CD95/Fas receptor (lpr-mice) or ligand (gld-mice) are protected in various animal models of acute neuronal damage, e.g. after ischemic stroke or spinal trauma $[8,9]$. Zuliani and coworkers provided first evidence that CD95/Fas-mediated "reverse signaling" might mediate neuroregeneration in the CNS [10]. They could show that CD95/Fas did not induce apoptosis in cortical and hippocampal neurons but rather controlled branching of these neurons in vitro. In

\footnotetext{
*These two authors contributed equally to this work Correspondence: Christoph P Beier

Tel: +49-941-941-3258; Fax: +49-941-941-3205

E-mail: Christoph.Beier@gmx.de
}

addition, the authors found a significant difference in the number of dendritic branches in mice deficient in CD95/ Fas and CD95L/FasL, suggesting an involvement of this mechanism during embryonic development. It is unknown whether CD95/Fas-controlled neuronal branching might be relevant in neurological diseases [10]. Because neuronal branching significantly contributes to neuroplasticity and therefore neurological recovery after acute neuronal damage, we speculated that CD95/Fas might be involved in the regeneration after traumatic brain injury (TBI). To test this hypothesis, we investigated the acute and chronic responses of CD95/Fas-deficient lpr-mice and wildtype control animals after controlled cortical impact (CCI).

CCI is a well-established animal model for TBI. It was performed using MRL-lpr mice and wildtype control animals with the same genetic background (composite genomic background: LG (75\%), AKR/J (12.6\%), C3H (12.1\%), C57BL/6 (0.3\%), Harlan UK Limited, Great Britain) as described previously (CCI: $2 \mathrm{~mm}$ flat-tip impounder; pressure: $25 \mathrm{kPa}$; depth: $1 \mathrm{~mm}$, impact duration: $100 \mathrm{~ms}$ ) [11]. Because MRL-lpr-mice develop systemic vasculitis at the age of 12 weeks [12], we used 6-8-week-old animals for the experiments. No signs of vasculitis were seen on hematoxylin/eosin-stained slides. To rule out a subconscious investigator bias, CCI, histological evaluation, and behavioral tests were performed "blinded".

The CCI lesion was placed over the motor cortex causing direct damage of motor cortex and indirect, time-dependent cell death of hippocampal neurons (Figure 1A). We used the beam walking test [13] to determine locomotor deficits and the Morris water maze test [14] to monitor the hippocampal function. In line with previous results, lpr-mice performed significantly better in both tests during the first $72 \mathrm{~h}$ after 
A
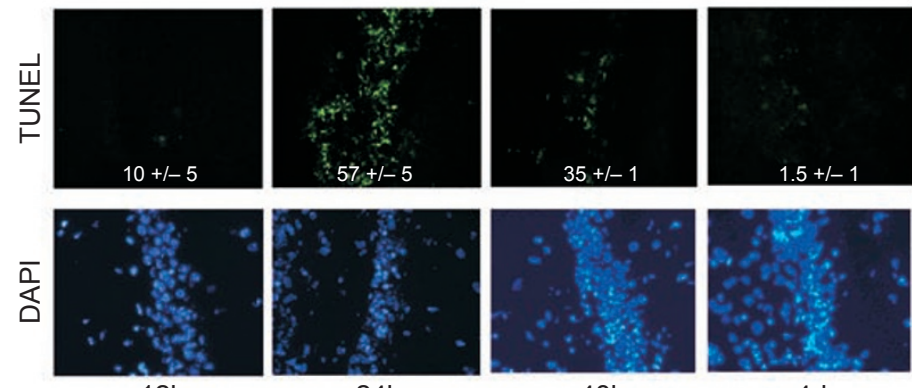

$24 \mathrm{~h}$

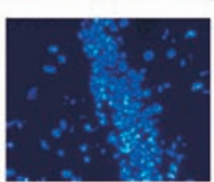

$48 \mathrm{~h}$

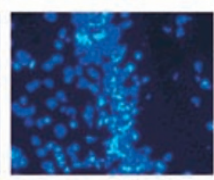

$4 d$

B

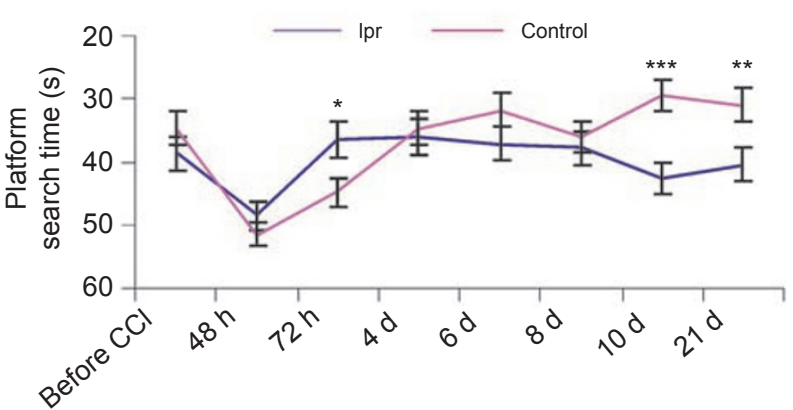

C

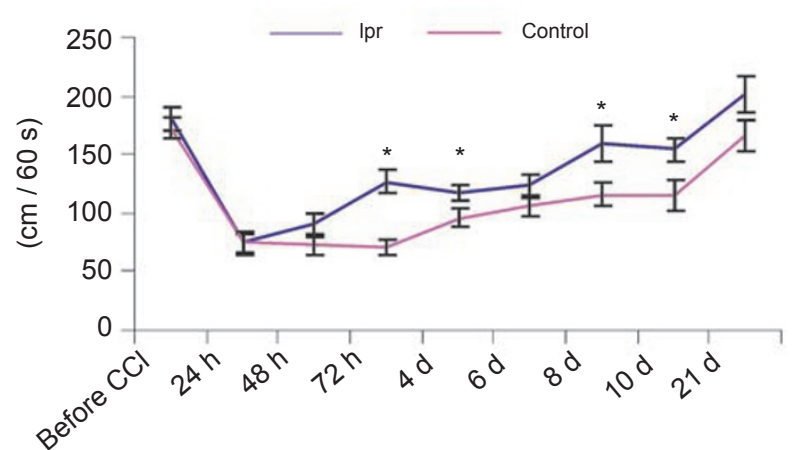

$\mathrm{D}$
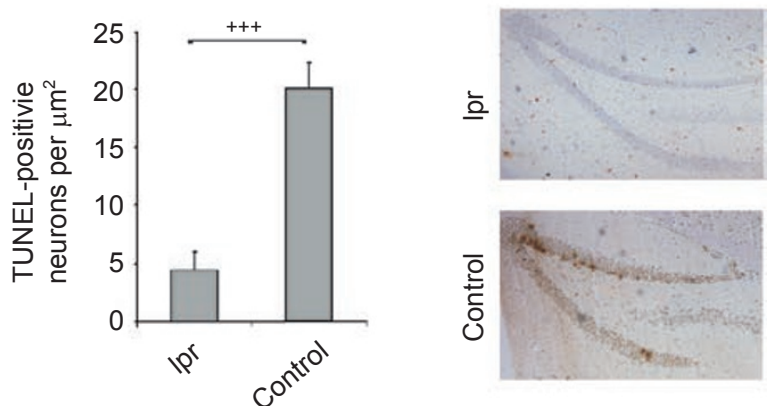

E
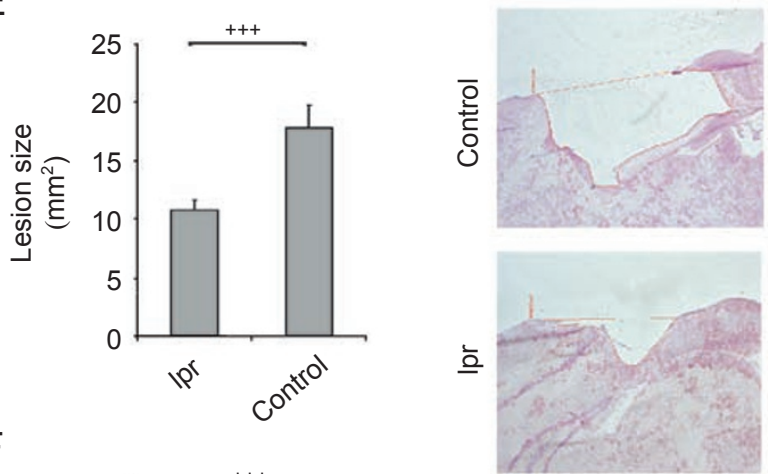

Figure 1 CD95/Fas mediates cognitive improvement after CCI. (A) Time course of indirectly damaged hippocampal neurons after CCI. Apoptotic neurons in the dentate gyrus of the hippocampus in wildtype animals were detected using the Apo-direct Kit (Chemi-

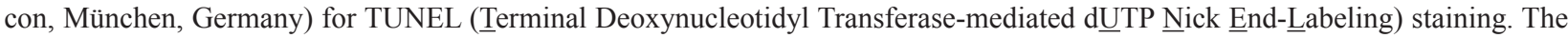
average number of apoptotic neurons in the dentate gyrus per slide at the time points indicated is given ( $\mathrm{n}=4$ animals). (B) Morris water maze test: Mice (lpr $\mathrm{n}=8$, control $\mathrm{n}=8$ ) learned to find the position of an invisible platform in a black water tank (diameter: $1.05 \mathrm{~m}$ with white signs for orientation) on 3 consecutive days before CCI. After CCI, the mice had to find the platform within 60 $\mathrm{s}$, where they were allowed to sit for $10 \mathrm{~s}$ ( 5 cycles per timepoint). The platform position remained unchanged during the entire experiment; the mouse was placed randomly in the tank. If the mouse did not find the platform within $60 \mathrm{~s}$, it was placed on the platform for $10 \mathrm{~s}$ to enable it to memorize its position. The average latency until the mice found the platform is shown. In all, 6 trials per time point and animal were performed. We did not conduct the Morris water maze test $24 \mathrm{~h}$ after CCI to avoid wound infections. (C) Beam walking test: The mouse was placed on an aluminum beam (diameter: $0.6 \mathrm{~cm}$, length: $60 \mathrm{~cm}$ ), from which it had to reach a large platform. The distance that the mouse covered within $60 \mathrm{~s}$ was determined. The test was repeated 4 times per time point. If a mouse fell down the beam or did not arrive within $60 \mathrm{~s}$ at the arrival platform, the distance until it fell down was measured. The mice learned to walk on the beam 6 times on 3 consecutive days before CCI. (D) At $24 \mathrm{~h}$ after CCI, lpr-mice had significantly fewer TUNEL-positive neurons in the dentate gyrus of the hippocampus compared with control animals. The number of TUNEL-positive neurons per $\mu \mathrm{m}^{2}$ is given. A total of 8 sections per animal $(\operatorname{lpr} n=5$, control $n=4)$ were analyzed. Representative pictures are shown. $(\mathbf{E}, \mathbf{F})$ The lesion volume and the size of the remaining brain tissue were determined after completion of the neurological testing 22 days after CCI $(\operatorname{lpr} \mathrm{n}=8$, control $\mathrm{n}=8)$. The average sizes of the lesion $(\mathbf{E})$ and the remaining cortical tissue $(\mathbf{F})$ are shown. In all, 5 sections per animal were assessed, representative lesions are shown $\left(* p<0.05,{ }^{*} p<0.01,{ }^{*} * p<0.001\right.$, one-way ANOVA; +++ $p<0.001$, Student's t-test; mean and SEM are given). 
the trauma (Figure 1B and 1C). The reduced neurological deficits were paralleled by a significantly reduced number of TUNEL-positive hippocampal neurons in lpr- as compared with wildtype mice $24 \mathrm{~h}$ after CCI (Figure 1D).

We further tested the mice until 21 days after the trauma. As compared with lpr-mice, wildtype mice had enduring and more pronounced locomotor deficits (Figure 1C) due to the direct damage of the motor cortex and subsequent larger lesions (Figure 1E) with less remaining brain tissue (Figure 1F). Notably, wildtype mice that had performed worse in the Morris water maze test directly after CCI improved their spatial memory function faster and better as compared with lpr-mice (Figure 1B).

The Morris water maze test determines the spatial memory function and the performance correlates with the integrity of hippocampal neurons that strongly express CD95/Fas $[5,14]$. The impaired improvement of the spatial memory function of lpr-mice after CCI therefore implies a functional relevance of the "death receptor" CD95/Fas expression on hippocampal neurons. Because neuronal branching is a major mediator for regeneration and subsequent functional recovery, it appears likely that CD95/Fas-mediated neuronal branching is involved in the cognitive improvement after CCI [10]. Our results therefore complement the in vitro data published by Zuliani et al. and suggest a functional relevance of CD95/Fas-mediated neuronal branching in this paradigmatic animal model.

CD95/Fas has a two-facet role in the pathogenesis of TBI. It is detrimental directly after the trauma and seems to become a mediator of regeneration by mediating neuronal branching in the later course of the disease. These conflicting functions are most likely mediated by regulatory proteins (e.g. LFG or c-FLIP) that are expressed in the hippocampus [6, 15] and regulated after TBI [15]. Therefore, targeting these proteins might be an excellent therapeutic approach for TBI aiming to inhibit apoptotic neuronal death initially and to promote neuronal recovery later on.

\section{Acknowledgments}

We wish to thank Marion Kubitza, Eva-Maria Stoerr, and Yesim Tevetoglu (Department of Neurosurgery, University of Regensburg) for their excellent technical assistance. Ralf Burger, Ana-Luisa Pina, and Ralf Rothoerl (Department of Neurosurgery, University of Regensburg) assisted with the animal experiments. This work was supported by a grant of the ReForm A program of the University of Regensburg to $\mathrm{CPB}$ and $\mathrm{DB}$.

\section{References}

1 Krammer PH. CD95's deadly mission in the immune system. Nature 2000; 407:789-795.

2 Barnhart BC, Legembre P, Pietras E, et al. CD95 ligand induces motility and invasiveness of apoptosis-resistant tumor cells. EMBO J 2004; 23:3175-3185.

3 Desbarats J, Birge RB, Mimouni-Rongy M, et al. Fas engagement induces neurite growth through ERK activation and p35 upregulation. Nat Cell Biol 2003; 5:118-125.

4 Bechmann I, Mor G, Nilsen J, et al. FasL (CD95L, Apo1L) is expressed in the normal rat and human brain: evidence for the existence of an immunological brain barrier. Glia 1999; 27:6274

5 Park C, Sakamaki K, Tachibana O, et al. Expression of fas antigen in the normal mouse brain. Biochem Biophys Res Commun 1998; 252:623-628.

6 Beier CP, Wischhusen J, Gleichmann M, et al. FasL (CD95L/ APO-1L) resistance of neurons mediated by phosphatidylinositol 3-kinase-Akt/protein kinase B-dependent expression of lifeguard/neuronal membrane protein 35. J Neurosci 2005; 25:6765-6774.

7 Cheema ZF, Wade SB, Sata M, et al. Fas/Apo [apoptosis]-1 and associated proteins in the differentiating cerebral cortex: induction of caspase-dependent cell death and activation of NF-kappaB. J Neurosci 1999; 19:1754-1770.

8 Demjen D, Klussmann S, Kleber S, et al. Neutralization of CD95 ligand promotes regeneration and functional recovery after spinal cord injury. Nat Med 2004; 10:389-395.

9 Martin-Villalba A, Herr I, Jeremias I, et al. CD95 ligand (FasL/APO-1L) and tumor necrosis factor-related apoptosis-inducing ligand mediate ischemia-induced apoptosis in neurons. J Neurosci 1999; 19:3809-3817.

10 Zuliani C, Kleber S, Klussmann S, et al. Control of neuronal branching by the death receptor CD95 (Fas/Apo-1). Cell Death Differ 2006; 13:31-40.

11 Woertgen C, Rothoerl RD, Brawanski A. Neuron-specific enolase serum levels after controlled cortical impact injury in the rat. J Neurotrauma 2001; 18:569-573.

12 Hewicker M, Trautwein G. Sequential study of vasculitis in MRL mice. Lab Anim 1987; 21:335-341.

13 Feeney DM, Boyeson MG, Linn RT, Murray HM, Dail WG. Responses to cortical injury: I. Methodology and local effects of contusions in the rat. Brain Res 1981; 211:67-77.

14 D'Hooge R, De Deyn PP. Applications of the Morris water maze in the study of learning and memory. Brain Res Brain Res Rev 2001; 36:60-90.

15 Hainsworth AH, Bermpohl D, Webb TE, et al. Expression of cellular FLICE inhibitory proteins (cFLIP) in normal and traumatic murine and human cerebral cortex. J Cereb Blood Flow Metab 2005; 25:1030-1040. 